\title{
Geometrical interpretation of long-time tails of first-passage time distributions in porous media with stagnant parts
}

\author{
Frank Wirner, ${ }^{1}$ Christian Scholz, ${ }^{1}$ and Clemens Bechinger ${ }^{1,2}$ \\ 12. Physikalisches Institut, Universität Stuttgart, Pfaffenwaldring 57, 70569 Stuttgart, Germany \\ ${ }^{2}$ Max-Planck-Institut für Intelligente Systeme, Heisenbergstraße 3, 70569 Stuttgart, Germany
}

(Received 31 January 2014; published 28 July 2014)

\begin{abstract}
Using a combined experimental-numerical approach, we study the first-passage time distributions (FPTD) of small particles in two-dimensional porous materials. The distributions in low-porosity structures show persistent long-time tails, which are independent of the Péclet number and therefore cannot be explained by the advectiondiffusion equation. Instead, our results suggest that these tails are caused by stagnant, i.e., quiescent areas where particles are trapped for some time. Comparison of measured FPTD with an analytical expression for the residence time of particles, which diffuse in confined regions and are able to escape through a small pore, yields good agreement with our data.
\end{abstract}

DOI: 10.1103/PhysRevE.90.013025

PACS number(s): 47.56.+r, 61.43.Gt, 47.55.Kf

\section{INTRODUCTION}

The transport of liquids through porous media plays an important role, e.g., for groundwater flow in aquifers and soils, tissue physiology, blood circulation through vein networks, and uptake of nutrients by plants, but it is also crucial for technical applications, such as bioremediation and enhanced oil recovery or filtering processes (for an overview, see Refs. [1-5]). The typically random structure of porous materials can lead to the formation of complex pathways along which the fluid's molecules are transported through such systems. The situation is even more difficult in low-porosity materials, which often contain stagnant parts where the flow velocity is extremely small or even zero. Once a molecule enters such a quiescent region, it can only escape by diffusion, independent of nearby advective currents. It is well known that stagnant parts (or dead-end pores) largely increase the retention times of molecules and thus have a strong effect on the fluid transport through porous materials. In addition, stagnant areas also lead to the efficient trapping of liquid in such media and thus have a large influence, e.g., on the exploitation efficiency of natural oil and gas reservoirs [6] but also for the efficiency of chromatographic columns and reactors [7].

Typically, the flow of tracer particles through a porous medium is described by the advection-diffusion equation (ADE), which reads as

$$
\frac{\partial c}{\partial t}+u \nabla c=D_{\mathrm{L}} \frac{\partial^{2} c}{\partial x^{2}}+D_{\mathrm{T}} \nabla_{\mathrm{T}}^{2} c .
$$

Here, $c(x, t)$ is the particle concentration, $u$ is the average particle velocity, and $D_{\mathrm{L}}$ and $D_{\mathrm{T}}$ are the longitudinal and transverse dispersion coefficients, respectively. Despite its simplicity, the ADE shows good agreement with experimentally measured concentration profiles in high-porosity materials with rather homogeneous flow fields [8]. However, the ADE fails to reproduce the slow decay of the particles' first-passage time distributions (FPTD) toward long times as observed in experiments and simulations [9-12]. According to Coats, Smith, and Baker, such long-time tails result from the exchange of particles between advective and stagnant regions [13-15]. Indeed, when Eq. (1) is expanded by an additional exchange term, it correctly describes the long-time tails of the FPTD observed for liquid flow through carbonate rocks, epoxies, and glass beads $[8,13,16,17]$. Rather than obtaining effective values for the stagnant volume fraction and a characteristic residence time of solutes in stagnant regions, however, such semiempirical models do not provide further structural information in porous materials.

In this paper, we experimentally study the flow properties of micron-sized colloidal tracer particles through quasi two-dimensional porous structures that contain well-defined stagnant areas. To obtain trajectories of sufficient duration, which are required to allow the particles to explore the interior of stagnant areas with large residence times, we applied a semiexperimental approach, where the experimentally determined velocity field was used as input information for a Langevin dynamics simulation. With this approach we create particle trajectories with maximum lengths of $10^{7} \mathrm{~s}$, which is far beyond typical time scales accessible in experiments. For low-porosity samples, we observed persistent long-time tails in the FPTD, which are independent of the Péclet number and that can be associated to temporary trapping of particles in stagnant regions. Comparison of these long-time tails with a theoretical prediction for the mean residence time of diffusive particles within a confined domain through a small exit pore yields quantitative agreement. This suggests a direct relationship between measured FPTD and the geometry of stagnant regions.

\section{EXPERIMENT}

To create porous samples with a well-defined morphology, we used a Boolean model where the structure is composed of randomly positioned, overlapping monodisperse circles (ROMC) with diameter $l=50 \mu \mathrm{m}$. Such structures were transferred to a transparent polydimethylsiloxane (PDMS) layer by soft lithography [18] and resulted in quasi two-dimensional porous samples with dimensions $6.5 \mu \mathrm{m} \times 3 \mathrm{~mm} \times 9 \mathrm{~mm}$ (height $\times$ width $\times$ length). A flow through such samples was induced by a hydrostatic pressure in the range of $\Delta P=$ $2-40 \mathrm{~Pa}$, which was created by two reservoirs with different water levels, which are connected to the sample's in- and outlet. The diameter of the reservoirs is about $2 \mathrm{~cm}$, therefore pressure 

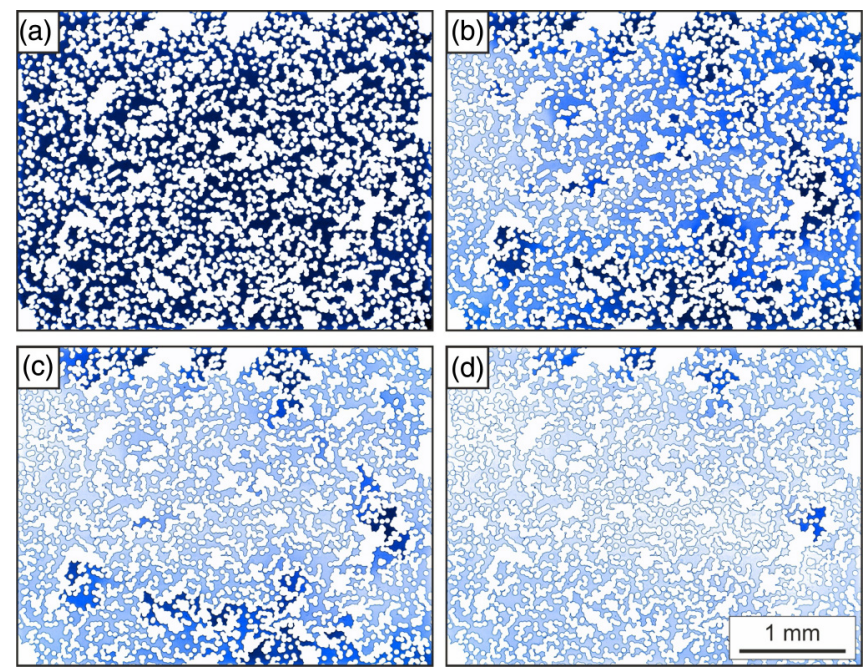

FIG. 1. (Color online) (a) Microscope image of a transparent porous structure made from PDMS, which is filled with dyed (blue) water. Afterwards, pure water is injected from the left with a flow velocity of about $6 \mu \mathrm{m} / \mathrm{s}$ and displaces the dyed water. Snapshots are shown after (b) $5 \mathrm{~min}$, (c) $10 \mathrm{~min}$, and (d) $33 \mathrm{~min}$. Even after more than $1 \mathrm{~h}$, dyed water remains trapped in the stagnant regions of the structure.

variations during experiments are negligible. For details of the sample preparation we refer to Refs. [19,20]. The flow field within the structure was determined by injection of a diluted (volume fraction $<10^{-3}$ ) suspension of fluorescent polystyrene particles with $1 \mu \mathrm{m}$ diameter. For this particle size, clogging within the structure and steric exclusion effects become negligible [21]. Particle trajectories were recorded by video microscopy with a frame rate of $5 \mathrm{fps}$ with $2240 \times$ 1724 pixel resolution at sub- $\mu \mathrm{m}$ precision using standard particle tracking algorithms [22].

For a first qualitative understanding of how stagnant areas affect the retention time of molecules, in Fig. 1 we show pictures of a sample with open porosity of $\phi_{\mathrm{o}}=0.400$ [23], which has been first saturated with dyed water (methylene blue) and then flushed by pure water from one side. At regions with high-flow velocity, this leads to the displacement of the dyed water within the first $10 \mathrm{~min}$. In contrast, the colored water remains trapped for much longer times in stagnant parts, where it remains even after $33 \mathrm{~min}$. This illustrates that time scales for reliable FPTD measurements in the presence of stagnant zones can be rather long.

For the quantitative determination of FPTD, we first measured the particle trajectories obtained at constant flow conditions (Péclet number Pe $\approx 130$ ) and then calculated the local velocity field [19]. In Fig. 2 we show the results in a grayscale (color) map obtained from samples with porosities $\phi_{\mathrm{o}}=0.900,0.582$, and 0.232 , respectively. The stagnant parts are identified by application of a velocity threshold, whose value is set to be about three orders of magnitude lower than the maximum velocity. We have confirmed that a variation of the threshold by a factor of 2 results only in minute changes of stagnant regions. With decreasing porosity, the velocity fields become increasingly inhomogeneous until only few principal pathways with high velocities (bright) are left (note the logarithmic grayscale). At the same time, an increasing area fraction appears where the velocity is extremely small, as typical for stagnant areas where the dynamics is dominated by diffusion. This spread in velocities is also reflected by the shape of particle trajectories, which are shown for $\phi_{\mathrm{o}}=0.232$ in Fig. 3(a). While the particle motion is rather directed in regions dominated by advective flow, a random walk behavior is found in stagnant areas. Although particles enter stagnant areas in our experiments, which were conducted over $30 \mathrm{~min}$, clearly these regions were only incompletely sampled (inset Fig. 3). Accordingly, FPTD obtained from such data are not meaningful and largely vary between measurements.

\section{SIMULATION OF PARTICLE TRAJECTORIES}

To solve the problem of poor statistics, we used the previously determined velocity field as input for an overdamped Langevin simulation. This allowed us to create particle trajectories with durations of up to $10^{7} \mathrm{~s}$ (in comparison to typical experimental time scales on the order of $5 \times 10^{3} \mathrm{~s}$ ). The particle displacement during the time interval $\Delta t$ is given by

$$
\mathbf{r}(t+\Delta t)=\mathbf{r}(t)+\mathbf{u}(\mathbf{r}) \Delta t+\xi(\Delta t),
$$
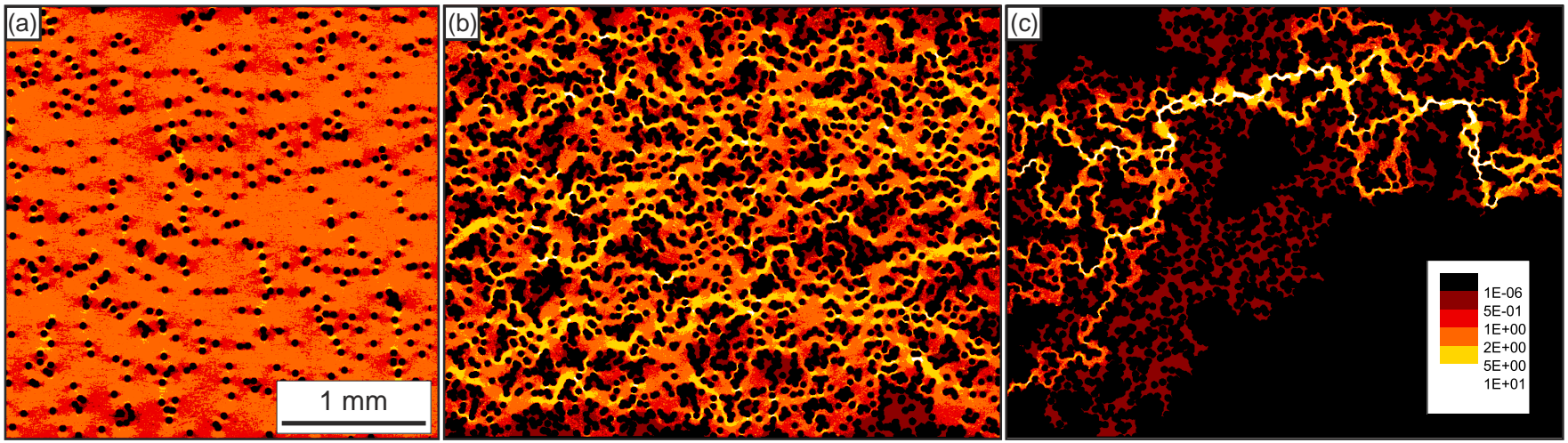

FIG. 2. (Color online) (a) Grayscale (color) maps of experimentally obtained velocity fields in porous ROMC structures with porosities (a) $\phi_{\mathrm{o}}=0.900$, (b) $\phi_{\mathrm{o}}=0.582$, and (c) $\phi_{\mathrm{o}}=0.232$. The plots show the local average velocity magnitude on a logarithmic scale in units of $\mu \mathrm{m} / \mathrm{s}$. With decreasing porosity, the velocity fields become more heterogeneous until only a few principal pathways for liquid flow remain, which are surrounded by large stagnant areas (dark). 

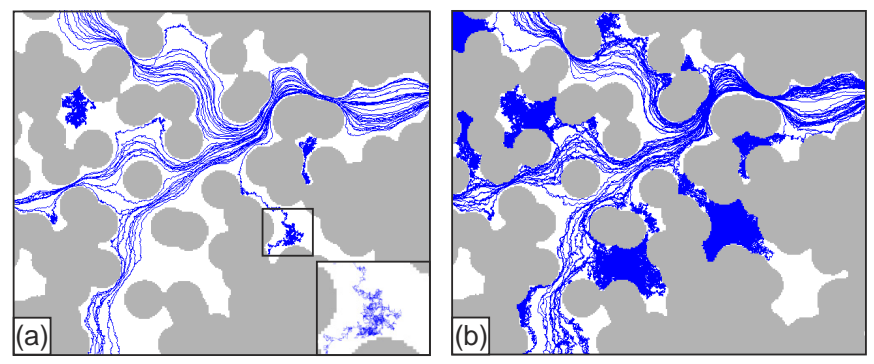

FIG. 3. (Color online) (a) Experimental particle trajectories obtained during $30 \mathrm{~min}$ inside a porous ROMC structure with $\phi_{\mathrm{o}}=$ 0.232 . The flow is from left to right. The inset shows a magnification of a diffusing particle in a stagnant region. (b) Corresponding particle trajectories obtained by simulations with observation time of $20 \mathrm{~h}$. Obviously, during this time the particles are able to explore much larger regions of the structure and in particular deeper parts of the stagnant areas.

with $\mathbf{u}(\mathbf{r})$ the experimentally determined particle velocity field and $\xi$ a random particle displacement due to thermal fluctuations being described by Gaussian distributed noise with zero mean and variance $4 D_{0} \Delta t$. The diffusion constant $D_{0}$ was set to the corresponding experimental value $D_{0}=4.3 \times$ $10^{-13} \mathrm{~m}^{2} / \mathrm{s}$. As time intervals we have chosen $\Delta t=40 \mathrm{~ms}$. At these values the largest particle displacements (corresponding to regions with the highest flow rates) do not exceed two particle diameters.

As an example, in Fig. 3(b) we plotted trajectories obtained from our Langevin simulations for the identical structure as in Fig. 3(a). Compared to the experimental data, the simulations cover a time interval that is about 40 times larger. In particular, the simulated trajectories are able to explore stagnant regions more thoroughly and thus provide realistic information about the structure of the sample. Typically more than 10000 particle trajectories with durations corresponding to more than $10^{7} \mathrm{~s}$ each were used for the FPTD as discussed below.

\section{RESULTS AND DISCUSSION}

\section{A. First-passage time distributions}

Figure 4 shows the FPTD obtained for samples with decreasing porosity $\phi_{\mathrm{o}}$ and for Péclet numbers $\mathrm{Pe}=$ $128,64,32,16$ [24]. For the structure with the highest porosity, $\phi_{\mathrm{o}}=0.900$, the corresponding FPTD are shifted toward larger first-passage times $T$ and also become considerably broadened with decreasing Péclet number [Fig. 4(a)]. The broadening is simply caused by the fact that at smaller Pe the particles' retention times inside the structure increase, and thus the overall dispersion during the passage of particles through the structure is enhanced. Since the velocity field of such high-porosity samples is rather homogeneous [see Fig. 2(a)], the ADE [Eq. (1)] should be applicable to describe the particle dynamics. Indeed the analytical expression of the FPTD as derived from the ADE is in perfect agreement with our measurements, as seen by the symbols in Fig. 4(a). Consequently, when the FPTD for different Péclet numbers are plotted on a logarithmic scale, they should only differ by a horizontal shift but otherwise should remain unchanged [3]. This is in agreement with our results [inset of Fig. 4(a)].
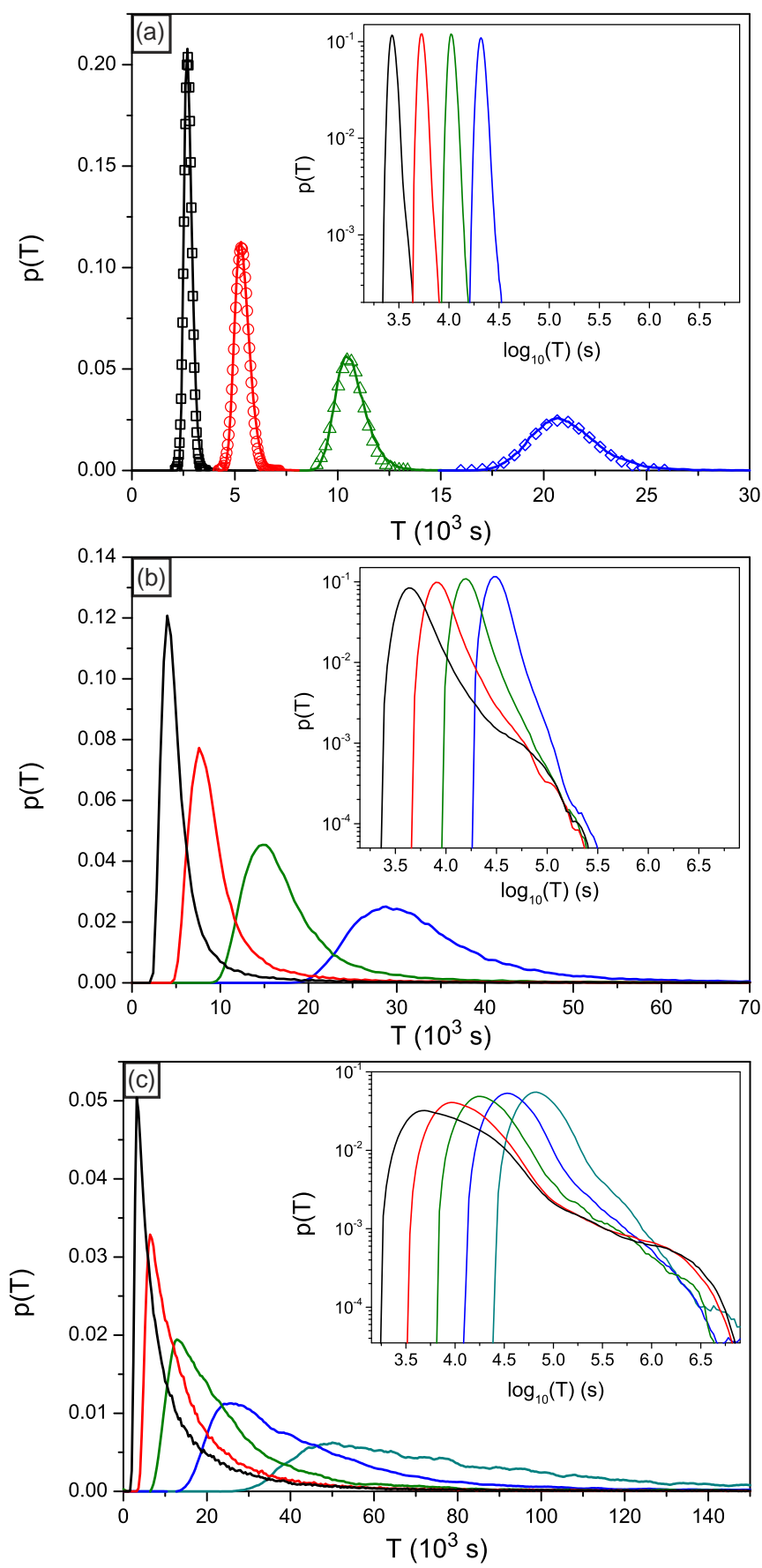

FIG. 4. (Color online) Measured FPTD for different Pe for three different structures (solid lines). The insets show the same data but on a logarithmic time scale. (a) Corresponds to $\phi_{\mathrm{o}}=0.900$, (b) to $\phi_{\mathrm{o}}=0.582$, and (c) to $\phi_{\mathrm{o}}=0.232$. The open symbols correspond to fits to the ADE. The highest Pe (black line) was 128 and each consecutive Pe decreases by a factor of two.

In contrast, the corresponding FPTD for samples with lower porosities $\phi_{0}=0.582$ and 0.232 are not correctly described by Eq. (1). In particular, the ADE is unable to reproduce the clearly visible long-time tails in Figs. 4(b) and 4(c). The occurrence of long-time tails is often explained by the inhomogeneities in the velocity field, which indeed increase with decreasing porosity [25]. This, however, is not 
in agreement with our experiments [see insets of Figs. 4(b) and 4(c)], which clearly show that the observed long-time tails are rather independent of the Péclet number. Instead, our observations suggest that these tails are caused by particles that were temporarily trapped in stagnant areas. Once a particle enters such a quiescent region, its dynamics is decoupled from the liquid flow through the sample until it escapes from such areas. During this time, its dynamics should be independent of the Péclet number, which indeed is in accordance with the long-time tails in Figs. 4(b) and 4(c).

\section{B. Comparison with theory}

Since the diffusive motion and escape probability of small particles in confined domains is important in many disciplines (e.g., in cell biology where the mean escape time is related to the time a molecule needs to hit a target binding site [26]). This problem has been theoretically studied in some detail $[27,28]$. In general, the mean residence time $\tau$ of a particle, which diffuses within a confined domain and can escape through an exit pore, depends on the starting position. It has been shown that the dependence on the distance $r$ between starting position and exit pore is rather weak, i.e., $\ln (r / a)$. where $a$ is the size of the exit pore [28]. For exit pores that are small compared to the total boundary of the domain, however, $\tau$ does not depend on the initial position except for a small boundary layer near the exit pore [29]. If the particle does not diffuse across the boundary layer, $\tau$ becomes rather small and therefore has only a very weak effect on the FPTD. Once a particle diffuses across this boundary layer, the escape time thus becomes a well-defined quantity that is given by

$$
\tau=\frac{A}{\pi D_{0}}\left[\ln \left(\frac{P}{d}\right)+1\right] .
$$

Here, $A$ and $P$ describe the area and perimeter of the domain and $d$ is the length of the exit pore. To obtain these quantities for our low-porosity samples, we first identified stagnant regions by application of a velocity threshold, as shown by the bright regions in Figs. 5(a) and 5(b). The contours of stagnant regions were obtained from the microscope images. To minimize discretization errors, we applied a marching squares algorithm [30] to determine $A, P$, and $d$. From the experimentally determined distribution of $A, P$, and $d$, Eq. (3) yields a distribution $N(\tau)$ of mean residence times [inset Fig. 5(c)] of the colloidal particles in the stagnant areas of our samples. We have validated Eq. (3) by numerical simulations, where we released diffusing particles at random positions within a stagnant part and calculated their escape times. Except for very small stagnant areas, where Eq. (3) might not be applicable, only small deviations have been found. In order to calculate first-passage times $T$ from such data, without taking into account all the intricacies of the transport process, one has to add the mean advection time $\tau_{\mathrm{A}}$, i.e., the time a particle needs to flow through the sample without being trapped in stagnant areas. The advection time corresponds approximately to the position of the maxima in the corresponding FPTD [31] as confirmed by comparison of first-passage times of particles, which never entered a stagnant region.

Figure 5(c) shows the first-passage times obtained from Eq. (3) for $\phi_{\mathrm{o}}=0.582$ and $\phi_{\mathrm{o}}=0.232$ for Pe $=128$ as solid

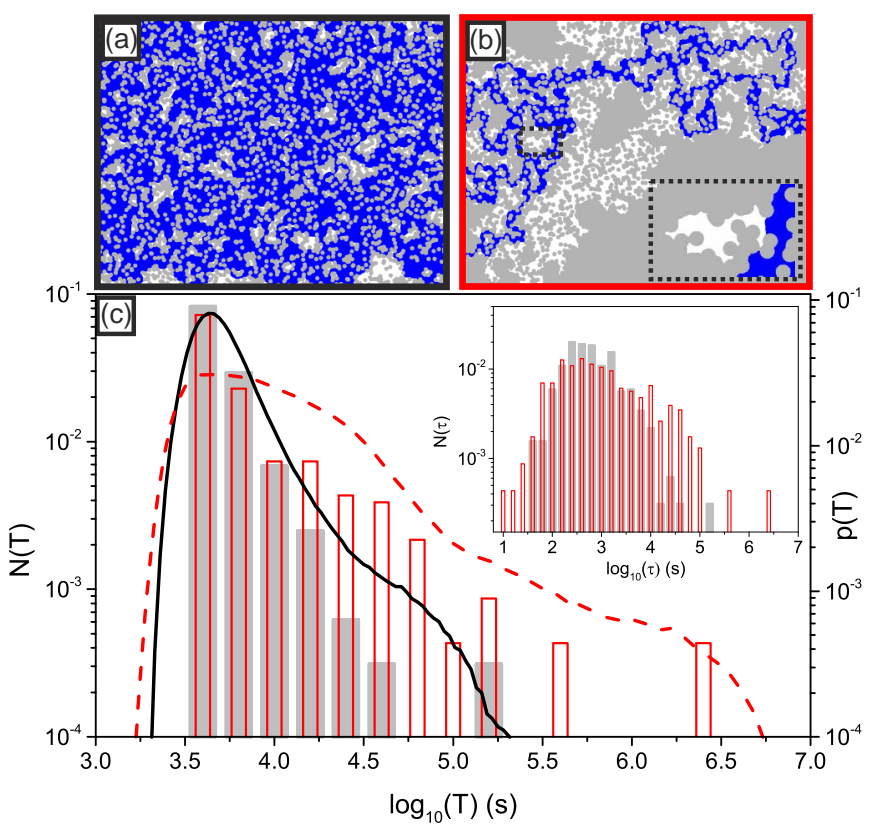

FIG. 5. (Color online) Flowing parts (dark blue) and stagnant parts (white) of (a) the structure with $\phi_{\mathrm{o}}=0.582$ and (b) $\phi_{\mathrm{o}}=0.232$. (c) Calculated FPTD due to stagnant parts for $\phi_{\mathrm{o}}=0.582$ (gray) filled bars and $\phi_{\mathrm{o}}=0.232$ (red) open bars. Solid (black) and dashed lines (red) show the measured FPTD for $\mathrm{Pe}=128$ for the structure with $\phi_{\mathrm{o}}=0.582$ and $\phi_{\mathrm{o}}=0.232$, respectively. (Inset c) Distribution of calculated mean residence times in stagnant parts.

(gray) and open (red) bars, respectively. For comparison we added the measured FPTD for these samples, taken from Figs. 4(b) and 4(c), as solid (black) and dashed (red) lines. Although the calculated and measured FPTD do only seem to moderately agree, it should be realized that in particular the largest values of the measured and calculated FPTD are almost identical. Given the simplicity of our assumptions and the fact that the retention times vary over more than four orders of magnitude, an accordance within a factor of two is remarkable. This good agreement suggests that the complex motion of particles through random porous media can be understood by superposition of a deterministic, flow-dependent advective contribution and a part which is independent of the Péclet number, the latter being caused by diffusion within stagnant regions. To appreciate how well our model compares to our experimental data, one also has to recall that our calculations assume that each particle becomes trapped only once within a stagnant zone. As will be shown, multiple trapping frequently occurs but only has a small effect on the long-time tails. In addition, the calculations are based on the mean residence time for a given stagnant area. Accordingly, such mean values cannot be expected to agree better with experimental data than the standard deviation of the escape time distribution, which equals its mean value.

\section{Influence of multiple trapping}

We also calculated from the trajectories the average number of stagnant zones $\bar{n}$ visited by a particle while flowing through the structure. In Fig. 6(a) we show this distribution for 

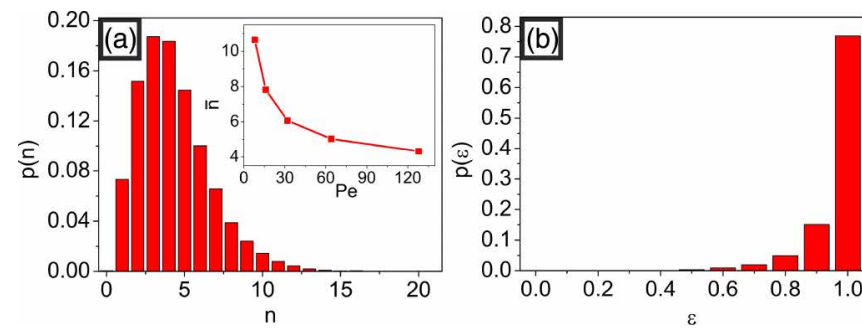

FIG. 6. (Color online) (a) Distribution of number of visited stagnant parts $n$, (b) fraction $\epsilon$ of time spent in largest stagnant part for $\phi_{\mathrm{o}}=0.232$ and $\mathrm{Pe}=128$. (Inset) Average number of visited stagnant zones $\bar{n}$ vs. Pe for $\phi_{\mathrm{o}}=0.232$.

$\phi_{o}=0.232$ and $\mathrm{Pe}=128$. From this it becomes obvious that multiple trapping events frequently occur. In addition, we also investigated the Pe-dependence of $\bar{n}$, which is shown as the inset of Fig. 6(a). With increasing Pe, $\bar{n}$ rapidly decreases from 10.6 to 4.4 .

To understand why multiple trapping in stagnant areas is still negligible in our samples for the long-time tails in the FPTD, it should be realized that the mean residence times of stagnant regions are not uniformly distributed [inset of Fig. 5(c)]. Therefore, long first-passage times require the trapping in the very few, but largest stagnant areas. This can be quantified by the parameter $\epsilon$, which is defined by the time spent in the stagnant part with the longest residence time relative to the total duration spent in all visited stagnant areas. Figure 6(b) shows the probability distribution of $\epsilon$ for $\mathrm{Pe}=128$ for first-passage times $T \geqslant 10^{5} \mathrm{~s}$, corresponding to the long-time tail in the FPTD. It can be clearly seen that at such large times trapping in a single, large stagnant area dominates the overall residence time. The dominance of a single stagnant area is also valid over the whole range of first-passage times (the mean value of $\epsilon$ amounts to about $80 \%$ ). This provides a posteriori a solid justification for the applicability of our simple model.

\section{CONCLUSIONS AND SUMMARY}

Since a corresponding expression for the residence time is also available for three-dimensional domains [29], our approach should apply to realistic porous media. Apart from inorganic materials this also includes biological systems, e.g., the brain tissue which can be modeled as a porous medium where the physiological response time of dissolved drugs depends on the morphological structure [26,32]. When the results of FPTD are combined with other morphological information obtained, e.g., by capillary pressure measurements [33] or adsorptiondesorption experiments [34], microscopic information about the structure of porous media and, in particular, the size and distribution of stagnant parts can be obtained.

In summary, we have measured FPTD of colloidal tracer particles in quasi two-dimensional porous model structures. At low porosities, we find long-time tails that are independent of the Péclet number and agree well with a simple expression for the mean residence time of particles, which diffuse within confined domains and escape through a small exit pore. This suggests a direct relationship between measured FPTD and the geometry of stagnant regions, which is crucial to understand the transport of fluids through porous media.

\section{ACKNOWLEDGMENTS}

We acknowledge fruitful discussions with David Holcman and Jakob Mehl.
[1] H. Brenner and D. A. Edwards, Macrotransport Processes (Butterworth-Heinemann, Oxford, UK, 1993).

[2] J. Bear, Dynamics of Fluids in Porous Media (Dover Publications, New York, 2013).

[3] M. Sahimi, Flow and Transport in Porous Media and Fractured Rock (Wiley, New York, 2012).

[4] G. Dagan and S. P. Neuman, Subsurface Flow and Transport: A Stochastic Approach (Cambridge University Press, Cambridge, 2005)

[5] D. Dan, C. Mueller, K. Chen, and J. A. Glazier, Phys. Rev. E 72, 041909 (2005).

[6] E. López, S. V. Buldyrev, N. V. Dokholyan, L. Goldmakher, S. Havlin, P. R. King, and H. E. Stanley, Phys. Rev. E 67, 056314 (2003).

[7] D. Kandhai, D. Hlushkou, A. G. Hoekstra, P. M. A. Sloot, H. Van As, and U. Tallarek, Phys. Rev. Lett. 88, 234501 (2002).

[8] E. Charlaix, J. Hulin, and T. Plona, Phys. Fluids 30, 1690 (1987).

[9] S. Silliman and E. Simpson, Water Resour. Res. 23, 1667 (1987).

[10] M. Levy and B. Berkowitz, J. Contam. Hydrol. 64, 203 (2003).

[11] A. Arya, T. Hewett, R. Larson, and L. Lake, SPE Reservoir Eng. 3, 139 (1988).
[12] B. Berkowitz and H. Scher, Phys. Rev. E 57, 5858 (1998).

[13] K. Coats and B. Smith, Old SPE J. 4, 73 (1964).

[14] L. Baker, Old SPE J. 17, 219 (1977).

[15] H. Deans, Old SPE J. 3, 49 (1963).

[16] G. Gist, A. Thompson, A. Katz, and R. Higgins, Phys. Fluids A: Fluid Dynam. 2, 1533 (1990).

[17] J.-C. Bacri, N. Rakotomalala, and D. Salin, Phys. Fluids A: Fluid Dynam. 2, 674 (1990).

[18] Y. Xia and G. M. Whitesides, Annu. Rev. Mater. Sci. 28, 153 (1998).

[19] C. Scholz, F. Wirner, Y. Li, and C. Bechinger, Exp. Fluids 53, 1327 (2012).

[20] C. Scholz, F. Wirner, J. Götz, U. Rüde, G. E. Schröder-Turk, K. Mecke, and C. Bechinger, Phys. Rev. Lett. 109, 264504 (2012).

[21] M. Auset and A. A. Keller, Water Resour. Res. 40, W03503 (2004).

[22] J. C. Crocker and D. G. Grier, J. Colloid Interface Sci. 179, 298 (1996).

[23] The open porosity $\phi_{\mathrm{o}}$ corresponds to the volume fraction in which fluid is present; i.e., it neglects closed cavities.

[24] Péclet numbers were determined by $\mathrm{Pe}=U l / D_{0}$, where $u$ is the particle velocity taken at the peak of the FPTD. 
[25] J. D. Hyman, P. K. Smolarkiewicz, and C. L. Winter, Phys. Rev. E 86, 056701 (2012).

[26] D. Holcman and Z. Schuss, Rep. Prog. Phys. 76, 074601 (2013).

[27] D. Holcman and Z. Schuss, J. Stat. Phys. 117, 975 (2004).

[28] O. Bénichou and R. Voituriez, Phys. Rev. Lett. 100, 168105 (2008).

[29] Z. Schuss, A. Singer, and D. Holcman, Proc. Natl. Acad. Sci. USA 104, 16098 (2007).
[30] G. E. Schröder-Turk, S. Kapfer, B. Breidenbach, C. Beisbart, and K. Mecke, J. Microsc. 238, 57 (2010).

[31] Practically all of the particles with these retention times also visit some very small stagnant areas.

[32] K. Khanafer and K. Vafai, Heat Mass Trans. 42, 939 (2006).

[33] A. Helba, M. Sahimi, L. Scriven, and H. Davis, SPE Res. Eng. 7, 123 (1992).

[34] N. Seaton, Chem. Eng. Sci. 46, 1895 (1991). 